\title{
El reto de enseñar una lengua extranjera sin recursos
}

\author{
Ana María Ramos GarcíA \\ Universidad de Granada
}

Recibido: 28 octubre 2009 / Aceptado: 14 enero 2010

ISSN: 1697-7467

\begin{abstract}
RESUMEN: La formación actual de los futuros maestros se centra, en gran medida, en la integración de las nuevas tecnologías en el aula de idiomas. Además, la invasión de las mismas en la vida diaria hace que nos hayamos vuelto «dependientes», de ahí que pareciese necesario comparar la situación actual de la enseñanza con la de hace unas cuantas décadas en nuestro país, para realizar una transición más comprensible hacia el contexto didáctico en lugares donde el acceso a las tecnologías es casi inexistente y los materiales y recursos disponibles son más bien escasos.

Palabras clave: enseñanza de lenguas extranjeras, TICs, características del profesorado, contexto de la enseñanza
\end{abstract}

\section{The Challenge of Teaching a Foreign Language without Resources}

\begin{abstract}
Nowadays, teacher training is quite influenced by ICTs and to some extent devoted to its use in the EFL classroom. Technology has invaded everyday life and we have got used to ICTs to such an extent that we do not remember how everything worked some years ago. That is why we compare the current teaching situation in Spain to that of several decades before. The aim of the comparison is to come closer to the teaching context in those places where technologies, teaching materials, and even basic resources are scarce.
\end{abstract}

Key words: TEFL, ICTs, FL teacher, teaching context

\section{INTRODUCCIÓN}

La integración de las nuevas tecnologías de la información y la comunicación (TICs) están cambiando nuestra forma de acceder a la información en general y por extensión están variando nuestra forma de proceder y comportarnos en la vida diaria, como demuestran los ejemplos que se explican a continuación:

* Somos menos pacientes: el uso de internet y la vasta cantidad de información a nuestro alcance en segundos hace que si una página web tarda un poco en abrirse la dejemos de lado y pasemos a la siguiente de la lista.

* Nos hemos vuelto más cómodos: el hecho de poder acceder a cualquier tipo de información desde casa a través del ordenador, hace que tanto alumnos como profesores utilicen principalmente fuentes «electrónicas» en lugar de visitar una biblioteca con la frecuencia de antaño. En el caso del alumnado el porcentaje de utilización de 
este tipo de fuentes es elevado, llegando a darse el caso de alumnos que cursando su último año de carrera no han visitado la biblioteca de su Facultad en ninguna ocasión.

* Estamos acostumbrados a localizar a una persona YA, o bien a través del móvil o bien a través de las múltiples opciones al alcance a través del ordenador (correo, chat, redes sociales, telefonía por internet etc.). Si la comunicación a través de todos estos medios no se llega a producir en el momento que esperamos nos invade una sensación de urgencia comparable a cuando nos olvidamos el móvil en casa.

* Todo es más veloz y vertiginoso en el mundo en el que vivimos y nos hemos acostumbrado de tal manera a esa inmediatez que ya forma parte de nuestras vidas, provocando ansiedad y haciendo que seamos menos receptivos hacia lo que pasa a nuestro alrededor y hacia las personas que nos rodean. Nos aislamos en nuestro mundo, rodeados por nuestras pertenencias y preferencias. El ejemplo más claro de este hecho es que ya no se camina por la calle o se viaja en autobús sin hacer otra cosa al mismo tiempo: hablamos por teléfono, escuchamos música, etc. En definitiva, nos abstraemos del mundo que nos rodea.

Las redes sociales están influenciando la forma de comunicarnos con los demás, especialmente entre los jóvenes. La información llega más lejos a más personas en menos tiempo. Ese es el factor esencial, ahorrar tiempo, hacer un esfuerzo menor para conseguir mejores resultados. La idea es buena, aunque ¿de verdad necesitamos ayuda para comunicarnos? Así reza el slogan de la red social más famosa a nivel internacional, Facebook: «te ayuda a comunicarte y compartir tu vida con las personas que conoces» (http://eses.facebook.com/).

Poco a poco las TICs se han ido integrando en nuestras tareas habituales, por lo que nos hemos acostumbrado a ellas y en mayor o menor medida dependemos de ellas (ordenador, internet, móvil, etc.) y se nos ha olvidado cómo se hacían las mismas cosas antes de su existencia. Una búsqueda de información, por ejemplo, resulta más rápida y sencilla que hace unos años y cuanto menos esfuerzo se invierte en una tarea menos huella dejará en nuestras vidas. La escasez o ausencia de papel, por ejemplo, ante la imposibilidad de anotar todo aquello que interesa, posibilita el ejercicio de la memoria y facilita que se recuerde más y mejor que en contextos más «favorables».

En líneas generales tenemos una menor capacidad de sacrificio que las generaciones precedentes y nos hemos vuelto más cómodos por las circunstancias en las que se desarrolla la vida diaria.

¿Qué ocurriría si de pronto no pudiésemos utilizar el ordenador, internet, el móvil o los omnipresentes reproductores portátiles a los que tan fácilmente nos hemos acostumbrado? Sería una vuelta a la realidad de nuestros mayores que tan lejana parece en estos momentos. Este es el contexto de la enseñanza en lugares remotos, sin comunicaciones con el exterior donde los niños también asisten a la escuela y deben aprender sin todas las facilidades que existen hoy por hoy en el mundo desarrollado. 


\section{Contexto}

\subsection{Contexto español actual}

Como se ha venido apuntando en páginas anteriores, la formación del profesorado de lengua extranjera se apoya en el uso de las nuevas tecnologías y se les enseña a los alumnos tanto a localizar recursos didácticos en la red como a crear sus propios materiales para el aula de Educación Primaria.

La aplicación de las nuevas tecnologías en el ámbito educativo ha dado lugar a la creación de centros TIC, escuelas en las cuales se emplean las nuevas tecnologías para mejorar el proceso de enseñanza-aprendizaje. Cuentan con conexión de banda ancha a internet y un ordenador por cada dos alumnos (vid. Fig. 1) que se utiliza como herramienta fundamental de aprendizaje. El profesorado dispone además de un ordenador portátil en el aula, proyector e impresoras locales y en red, y también de escáneres, normalmente ubicados en los departamentos ${ }^{1}$.

El empleo de las nuevas tecnologías requiere un cambio en la metodología docente, por ello el profesorado recibe formación específica para integrar de forma efectiva las TICs en el proceso de enseñanza.

Estos procesos de reciclaje y formación continua del profesorado son extensivos al campo de lenguas extranjeras ya que el contexto en el que desarrolla la docencia ha cambiado mucho:

La diversidad lingüística, étnica, social y cultural a la que tienen que hacer frente en las aulas [...] está propiciando una revisión y actualización de la formación recibida por el profesorado para que puedan responder de manera adecuada a las demandas profesionales que recaen sobre ellos. (Morales et al. 2000; 190)

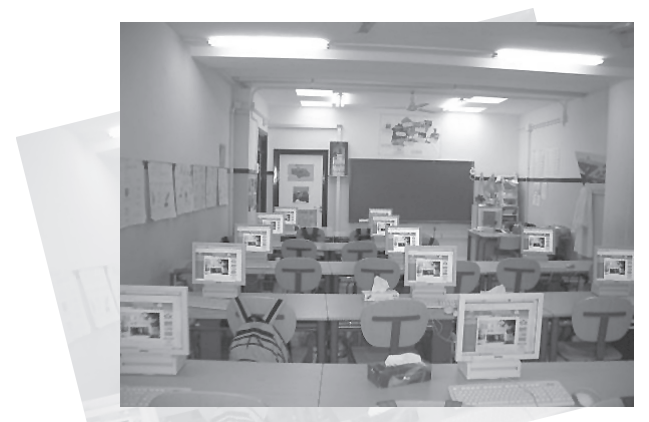

Fig. 1: CEIP E.Tierno Galván, La Zubia (Granada)²

\footnotetext{
${ }^{1}$ http://www.juntadeandalucia.es/averroes/sanwalabonso/centro_tic.htm consultado el 27 de septiembre de 2009.

${ }^{2}$ Extraída de http://www.juntadeandalucia.es/averroes/centros-tic/04005818/helvia/sitio/index.cgi?wid_seccion= 4\&wid_item=13 el 27 de septiembre de 2009.
} 


\subsection{Contexto español de hace cuarenta años}

El contexto educativo de países (o algunas zonas dentro de ellos) como Gahna, Kenia, Mongolia, Ecuador, Colombia o Nicaragua, entre otros, es bien diferente ya que se reduce únicamente al profesor, al alumnado y a los materiales con los que cuenten para apoyar la docencia, aunque generalmente la característica principal es la falta de ellos.

Este contexto es equiparable, a grandes rasgos, a la situación española de hace unos cuarenta años, cuando la mayoría de las escuelas estaban situadas en zonas rurales y en muchos casos de difícil acceso. Había un único maestro para todos los niños de la zona, independientemente del curso o edad del alumnado.

El contexto didáctico, era, por tanto, muy diferente: hasta 90 alumnos (en algún caso más) de cursos diferentes que tenían que compartir el aula al mismo tiempo y con el mismo maestro.

El maestro, evidentemente, debía gestionar el tiempo de manera efectiva para que todos pudiesen trabajar a un tiempo y a la vez explicar algo concreto a uno de los subgrupos de la clase. Vivía, generalmente, en la casa-escuela (si contaba con ella) o en algún lugar cercano. Los transportes eran escasos y lentos y las escuelas estaban en la mayoría de los casos a kilómetros de distancia de donde alcanzaba a llegar el transporte. Algunos alumnos debían caminar bastante para llegar a la escuela y la mayoría de ellos ayudaba en casa en las tareas de labranza y cuidado del ganado antes y después de ir a la escuela.

\subsection{Contexto actual en zonas remotas sin recursos}

La situación en cualquiera de los países antes mencionados es bastante similar en líneas generales, aunque llega a ser peor en muchos casos: la mayoría de los alumnos no cuentan con transporte escolar y deben caminar hasta diez kilómetros o más para ir al colegio, sin comida ni bebida hasta que vuelven a casa, esto es, veinte kilómetros en total más la jornada escolar. Estas condiciones tan precarias se repiten con demasiada frecuencia, llegando a haber casos espeluznantes como el de la aldea Los Piños en plena selva Colombiana, que se describe a continuación.

Esta remota aldea colombiana se halla en la selva y cuenta con escasas conexiones con la civilización por motivos económicos. Dada su ubicación únicamente se puede acceder al exterior de dos maneras: la primera, a través de una larga y peligrosa caminata de dos horas campo a través o, la segunda, por medio de un sistema de tirolina doble, una en sentido de ida y otra de regreso para poder cruzar un cañón. El gobierno no construye un puente en la zona porque considera que el número de habitantes es bajo para justificar el gasto, así que los niños deben utilizar este sistema para asistir al colegio todos los días. Deben llevar una polea y un freno (hecho con un palo) además de una cuerda que hace la función de arnés. Nadie sabe el límite de carga de este rudimentario sistema de transporte y para garantizar la seguridad de los niños más pequeños, estos son transportados por hermanos mayores en un saco $^{3}$.

${ }^{3}$ Vídeo disponible en: http://www.noob.us/miscellaneous/kids-ride-a-zip-line-to-go-to-school/ consultado el 27 de septiembre de 2009. 
Es evidente que estos niños están motivados y tienen ganas de aprender porque en algunos casos les da miedo tener que utilizar este «transporte escolar». Así que su proceso de aprendizaje será más exitoso pues «el factor más importante para tener éxito en la adquisición de una segunda lengua es la motivación» (Suárez Rodríguez, 2009: 8).

\section{Condiciones del aula de LE}

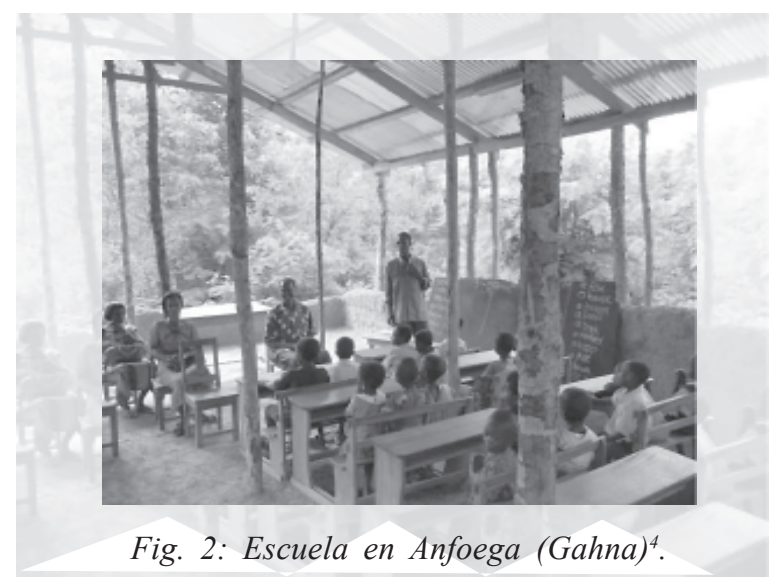

Normalmente la docencia se desarrolla basándose en la utilización de un libro de texto. El profesorado con poca experiencia docente suele sentirse más seguro al poder seguir las indicaciones del mismo, alejándose en pocas ocasiones de lo allí mencionado. El papel del libro de texto y sus materiales adicionales se ha sobrevalorado en exceso, llegando a convertirse en una especie de Biblia, pero en contextos menos favorecidos, lo que suele ocurrir es que no hay suficientes ejemplares para todos los alumnos, no se cuenta con la edición especial para el profesor, suelen estar bastante anticuados y tienen poco o nada que ver con la realidad existencial del alumnado y las fotocopias resultan caras y prácticamente inaccesibles (Chávez, 2006: 33).

No hay cintas de casete ni CDs para ejercitar la comprensión oral, pero aunque los hubiera, probablemente no habría ningún aparato para hacerlos sonar.

En aquellos lugares en los que hay ordenadores, estos son bastante lentos, las impresoras no proliferan y el papel resulta un bien escaso y de elevado coste económico.

Las conexiones a internet, donde las hay, no funcionan regularmente pues las instalaciones no son adecuadas.

El profesorado de Lengua Extranjera no suele ser especialista, «a large number of English teachers are currently teaching without appropriate professional qualifications and training» (Chávez, 2007: 27-28). Esto provoca que el uso de la LE en el aula sea escaso (por falta de seguridad y/o conocimientos necesarios para desarrollar una clase en inglés) y que sea nece-

${ }^{4}$ Extraída de http://pwathne.dk/pictures.html el 27 de septiembre de 2009. 
saria la creación y puesta en marcha de programas de mejora como, por ejemplo, el «Saturday English Program» que se está desarrollando en la Universidad Nacional Autónoma de León en Nicaragua (Chávez, 2006: 27).

La descripción realizada por Chávez (2006) sobre la situación del profesorado de LE de Secundaria en Nicaragua es descorazonadora, pues argumenta que su ínfimo salario no les permite cubrir sus necesidades más básicas, así que se ven obligados a trabajar el doble o triple de la jornada laboral normal para poder subsistir (Chávez, 2006: 33). El cansancio y la ratio profesor-alumno hacen que la motivación sea escasa entre los docentes.

\section{Práctica docente}

Tras la observación de la imagen (Fig. 2) en un contexto sociocultural como el que se ha venido describiendo en los apartados precedentes, la primera idea que surge es que resultaría bastante difícil enfrentarse a la enseñanza en esas condiciones.

Si se sigue observando la imagen más detalladamente y desde el punto de vista de un docente, especialmente si este es joven y con poca experiencia, se hace evidente la pobreza y desnudez del aula. No hay materiales, ni libros, ni siquiera paredes donde colgar un mural, así que la pregunta que nos plantearíamos sería ¿qué enseñar? Obviamente la respuesta viene dada por el contexto, edad y necesidades del alumnado, así que una vez solventada la cuestión, la reflexión continuaría hacia ¿cómo enseñar? ya que en la formación de los futuros maestros, en nuestro contexto, se insiste con frecuencia en el apoyo de la enseñanza en una miríada de materiales adicionales para facilitar la comprensión y aprendizaje del alumnado, a través de la utilización de las nuevas tecnologías como medio de obtención de información y materiales y como medio de exposición de los mismos.

La situación, como se viene puntualizando, es bien diferente, ni siquiera podemos apoyarnos en el libro de texto, ese elemento que aporta tanta seguridad al profesorado en sus primeros años de ejercicio docente.

Nos encontramos, por tanto, la enseñanza en estado puro: el docente, el alumnado y una falta de recursos abrumadora, que se suelen reducir a una pizarra y una tiza y, si la situación es un poco más favorable, a los cuadernos de los alumnos, si bien, como se ha puntualizado anteriormente, el papel es un recurso caro y difícil de conseguir.

Obviamente el éxito del proceso de enseñanza-aprendizaje en este contexto recae, más que en ningún otro, en la capacidad del maestro para enseñar en esas condiciones.

Es necesario ser consciente de que para enseñar no hacen falta una gran cantidad de recursos materiales, simplemente hay que ser creativo y buen profesor.

Para definir lo que se debe enseñar, en primer lugar, es necesario conocer el contexto legal y social del lugar en el que se pretende desarrollar la docencia. La legislación educativa vigente nos aportará información importante sobre lo que deben aprender nuestros alumnos y cuándo deben hacerlo. Los conocimientos sobre el contexto social son importantes para adaptar esos contenidos a la realidad en la que viven los alumnos. Por ejemplo, si el lugar en el que nos encontramos no cuenta con agua corriente, resultaría más útil enseñar a los alumnos cómo decir «transportar agua» en lugar de «grifo».

La adaptación de los contenidos al medio en el que vive el alumnado produce mejores resultados en el aprendizaje: 
Children make sense of the world and of language through the context they find themselves in. Children pay far more attention to the whole situation than to language, and the younger they are, the truer this is. (Read, 2007: 8)

Un ejemplo de ello es el Método Sanabria, método de alfabetización onomatopéyico que consistía en asociar cada letra con un gesto para lograr fijar en la mente la grafía y el fonema correspondiente. Cada letra del abecedario era un personaje al que le sucedía algo narrado en forma de cuento. El maestro hacía un dibujo del personaje en la pizarra y luego para recordarlo iba borrando las partes del cuerpo hasta que quedaba únicamente la letra en cuestión. A través de la narración de historias los niños recordaban fácilmente las letras, además del gesto asociado a ellas. Las narraciones se situaban en el medio rural, el contexto habitual del alumnado del inventor del método: Matías Martín Sanabria.

\subsection{Características del profesor de Lengua Extranjera}

Las características del buen profesor de Lengua Extranjera, según Madrid (2005:227) son las siguientes:

* Explica con claridad

* Aumenta el interés y motivación

* Pronuncia bien

* Habla con fluidez

* Se adapta a las necesidades y características personales del alumnado

* Es justo al evaluar

* Es activo y fomenta la participación

* Crea un ambiente cómodo en clase

Para manejar una clase de forma eficaz y positiva el maestro debe «tomar conciencia de los comportamientos que su actitud puede inducir en sus alumnos» y también «permanecer vigilante a sus propias reacciones ante algunos comportamientos de alumnos» (Bailly, 2005: 167).

La comunicación no verbal es importantísima en cualquier contexto educativo, pero principalmente en este en el que no hay elementos externos como pantallas, murales o dibujos, que puedan distraer tan fácilmente la atención del alumnado.

Comunicamos inconscientemente con nuestras actitudes y expresiones ya que «el lenguaje no verbal funciona a pesar de que no seamos conscientes de él» (Albaladejo, 2007: 29). Por ello es necesario controlar qué transmiten nuestros gestos para que sean acordes con lo que decimos y además, utilizarlos para gestionar la clase: aclarar significados sin traducir, animar al alumnado a participar, demostrar que se presta atención y que lo que nos dicen es importante.

Albaladejo (2007: 27) da cuenta de una investigación cuantitativa de la comunicación no verbal en la comunicación citando a Mehrabian (1972) que determinaba que:

la parte estrictamente verbal de la comunicación, entendida como vocabulario y la gramática, ¡solamente aporta el 7\% de la información transmitida! El 93\% restante se distribuye entre elementos corporales (55\%), como el gesto, el tacto o la mirada, $\mathrm{y}$, un $38 \%$ de información transmitida a través de la voz (entonación, ritmo, volumen, etc.), otro dato sorprendente. 
Los aspectos esenciales para que el aprendizaje de la LE en el aula sea efectivo son, entre otros, los siguientes:

* Es necesario realizar un uso frecuente de la LE, especialmente para las rutinas habituales en la clase y el vocabulario básico de uso frecuente, porque probablemente sea el único contacto con la LE que tengan los alumnos.

* Fomentar la participación en LE.

* Enseñar gramática en contexto, no como fin único de la enseñanza.

* Premiar cualquier esfuerzo realizado por el alumnado (independientemente del resultado).

* Propiciar la comunicación entre iguales, tanto en parejas como en grupo.

* Hacer de las representaciones (narraciones, diálogos, etc.) algo habitual.

\section{Actividades}

Los niños aprenden de múltiples formas: observando, escuchando, imitando o haciendo cosas (Slattery y Willis, 2001: 4) pero es necesario recordar que «las lenguas se aprenden fundamentalmente a través de la imitación» (Suárez Rodríguez, 2009: 8).

Las consideraciones principales a tener en cuenta si se trabaja con niños de hasta 7 años según Stattery y Willis (2001: 4) son las siguientes:

* Introducir el idioma despacio a través de actividades divertidas

* Usar muchos gestos, acciones, movimientos y expresiones

* Fomentar la seguridad repitiendo actividades ya conocidas

* Repetir en LE lo que dicen en su LM

* Contar cuentos, supliendo la falta de materiales de apoyo (fotos y dibujos)

$\mathrm{Si}$, en cambio se trabaja con niños de entre siete y doce años (Slattery y Willis, 2001: 5) es recomendable tener en cuenta lo especificado para alumnos más pequeños y además:

* Explicar al principio de curso por qué se usa el inglés durante la clase

* Enseñar frases útiles para preguntar sobre el uso de la lengua, por ejemplo: «What's... in English?».

* Planificar con antelación la clase.

* Hablar sobre experiencias personales: les encanta saber cosas de su maestro, qué le gusta, qué le divierte, historias personales, etc.

* Pasarlo bien

A continuación se proponen actividades de carácter general, siguiendo a Slattery y Willis (2000) y Read (2007) adaptables a múltiples situaciones didácticas para las cuales no hace falta contar con materiales adicionales, simplemente con los conocimientos y habilidades del maestro.

1. Listen and identify:

1.1. Vocabulario básico: (todo lo que se pueda ver en clase y alrededor)

1.2. Grammatical awareness (singular/plural, pronouns) 
2. Listen and do

2.1. Respuesta física total

2.1.1. Follow the leader

2.1.2. Repaso de vocabulario (colours \& clothes)

2.1.3. Rutinas:

* what do we do when...

* Acciones (clap your hands, slap your legs,...)

* Pointing rhymes:

Point to the ceiling,

point to the floor,

clap your hands together,

$1,2,3$,

now sit down and look at me

(adaptada de Slattery y Willis, 2001: 25)

* Rearranging the class

3. Listen and perform (miming): también con canciones y rimas

4. Listening and responding games

4.1. Right or wrong

4.2. Memory games

5. Listen and draw / colour (si hay papel)

6. Speaking:

6.1. Talking about families

7. Cuentos: ¡no hay libros!

7.1. Historias personales del profesor

8. Juegos:

8.1. Acciones: Simon says

8.2. Bingo

8.3. Vocabulario

8.3.1. adivinar palabras ya estudiadas

8.3.2. memoria

8.4. I spy with my little eye (veo, veo)

9. Canciones: no hay reproductores, así que... ¡a cantar! Elegir canciones que permitan acompañarlas con mímica, del tipo «Head, shoulders, knees and toes».

Las canciones ayudan a ejercitar la memoria y la concentración, especialmente si se varía el volumen y se pide a los alumnos que canten en voz baja, en tono normal o muy alto.

by building on children's pleasure in rhythm and sounds, they engage children in responding to and producing language in ways which develop their confidence and self-esteem and lead to a sense of achievement and success (Read, 2007:182).

9.1. Temática: saludos, despedidas, cosas de clase, números, acciones, partes del cuerpo, animales, animales y sus crías, canciones que narran historias, etc. (Read, 2007:185). 
9.2. Eco: repetir lo que canta el profesor. Una vez se hayan aprendido la canción, ellos pueden dirigir el juego. (Read, 2007: 202).

9.3. Canon: dividir la clase en grupos y que cada uno cante un fragmento y los otros respondan, o que vayan repitiendo la letra comenzando en momentos diferentes. (Read, 2007: 203).

9.4. Omitir o añadir palabras nuevas en la letra ya conocida (Read, 2007: 203)

9.5. Distinguir las palabras que riman en una canción

10. Representaciones

10.1. Narraciones

10.2. Diálogos...etc.

Resulta esencial variar el tipo de actividades con frecuencia y no limitarse a repetir incansablemente las mismas, pues los alumnos se cansarían de hacer siempre lo mismo, disminuyendo así su motivación e implicación en la clase considerablemente: «expand your range of interactive activities. Don't fall back on the same lame handful each time (like hangman and bingo)» (Jenkins, s.d.: 86)

\section{Evaluación}

$\mathrm{Si}$, como se ha repetido en varias ocasiones a lo largo de estas páginas, no tenemos un fácil acceso a recursos como el papel, difícilmente podremos elaborar un examen escrito para los alumnos, aunque sí se podrían realizar pruebas orales.

La evaluación debería llevarse a cabo, por tanto, a través de una observación detallada de los progresos realizados por los alumnos en la clase, llevando un registro individualizado sobre cada uno de ellos.

\section{Conclusión}

Como se ha demostrado a través de este artículo, la ausencia de los recursos más básicos en el aula podría parecer, a priori, una limitación considerable en el ejercicio de la docencia, pero en realidad se trata de una oportunidad excelente para desarrollar y demostrar las competencias del maestro como tal. Sería un buen ejercicio práctico de formación para los futuros docentes imaginarse por un día que se encuentran en estas condiciones tan precarias y desarrollar su tarea docente sin recursos, así serían conscientes de las dificultades que ello conlleva y mejorarían considerablemente su calidad como docentes.

No es mejor maestro el que más sabe, ni siquiera el que más enseña, sino el que mejor educa, el que tiene el raro don de hacer hombres dueños de sí y de sus facultades y acciones. No el que más trabaja, sino el que más hace trabajar con más gusto a los alumnos. No es el que más discurre, sino el que más y mejor enseña a pensar. No es el que mejor diserta, sino el que mejor dialoga con sus escolares. No es el que más eleva, sino el que más allana y abaja para ascender con los discípulos hasta ponerlos a su altura para descubrir nuevos horizontes. (Manjón, 1923). 


\section{Bibliografía}

Albaladejo Mur, M. (2007). La comunicación más allá de las palabras. Qué comunicamos cuando creemos que no comunicamos. Barcelona: Graó.

Bailly, B. (2005). Enseñar: una cuestión de personalidades. Barcelona: Ediciones CEAC.

Brewster, J.; Ellis, G. y Girard, D. (2002). The Primary English Teacher's Guide. Harlow: Pearson Education.

Chávez, E. (2006). «In-service teachers' beliefs, perceptions and knowledge in the Nicaraguan EFL context», en Encuentro, 16: 27-39.

Jenkins, J. (s.d.). TEFL Uncovered. How to Teach your Way Abroad with TEFL. Extraído de http:/ /www.kent.ac.uk/careers/docs/tefl-uncovered-low.pdf el 27 de septiembre de 2009.

Madrid, D. (1996). «The FL Teacher», en McLaren, N. y Madrid, D. (eds.), A Handbook for TFL. Alcoy: Marfil, 107-128.

Madrid, D. y McLaren, N. (eds.) (2004). TEFL in Primary Education. Granada: Editorial Universidad de Granada.

Madrid, D. y McLaren, N. (eds.) (2005). TEFL in Secondary Education. Granada: Editorial Universidad de Granada.

Manjón, A. (1923). El maestro mirando hacia fuera o de dentro a fuera. Libro IV. Maestros didácticos y antididácticos. Madrid: Revista de Archivos, Bibliotecas y Museos.

Morales Gálvez, C.; Arrimadas Gómez, I.; Ramírez Nueda, E.; López Gayarre, A. y Ocaña Villuendas, L. (2000). La enseñanza de las lenguas extranjeras en España. Madrid: Ministerio de Educación y Ciencia. Secretaría General Técnica.

Read, C. (2007). 500 Activities for the Primary Classroom. Oxford: Macmillan Education.

Slattery, M. \& Willis, J. (2001). English for Primary Teachers. Oxford: Oxford University Press.

Suárez Rodríguez, I. M. (2009) «Recursos didácticos para la enseñanza de inglés, aplicables al alumnado con deficiencia visual» Gibralfaro, 59. Obtenido de http://www.gibralfaro.uma.es/ numerospub/numero_59.htm el 27 de septiembre de 2009. 
